\title{
Heavy Fragments Identification Using Energy Loss Method in the STS Detector of the CBM Experiment
}

\author{
Olga Derenovskaya ${ }^{1, \star}$, Victor Ivanov ${ }^{1,2}$, lorii Vassiliev ${ }^{3,4}$, and Yurii Russov ${ }^{2}$ \\ ${ }^{1}$ Laboratory of Information Technologies, Joint Institute for Nuclear Research, Dubna, Russia \\ ${ }^{2}$ National Research Nuclear University "MEPhl", Moscow, Russia \\ ${ }^{3}$ Gesellschaft für Schwerionenforschung $\mathrm{mbH}$, GSI, Darmstadt, Germany \\ ${ }^{4}$ Goethe-Universität, Frankfurt am Main, Germany
}

\begin{abstract}
The current paper presents the possibility of heavy fragments identification using energy loss method and $\omega_{n}^{k}$ criterion in the STS detector of the CBM experiment.
\end{abstract}

\section{Introduction}

The Compressed Baryonic Matter (CBM) Collaboration [1] conducts dedicated heavy-ion experiments to investigate the properties of highly compressed baryonic matter as is produced in nucleusnucleus collisions at the Facility for Antiproton and Ion Research (FAIR) in Darmstadt, Germany.

The electron-hadron configuration of the CBM experimental setup is shown in Fig. 1.

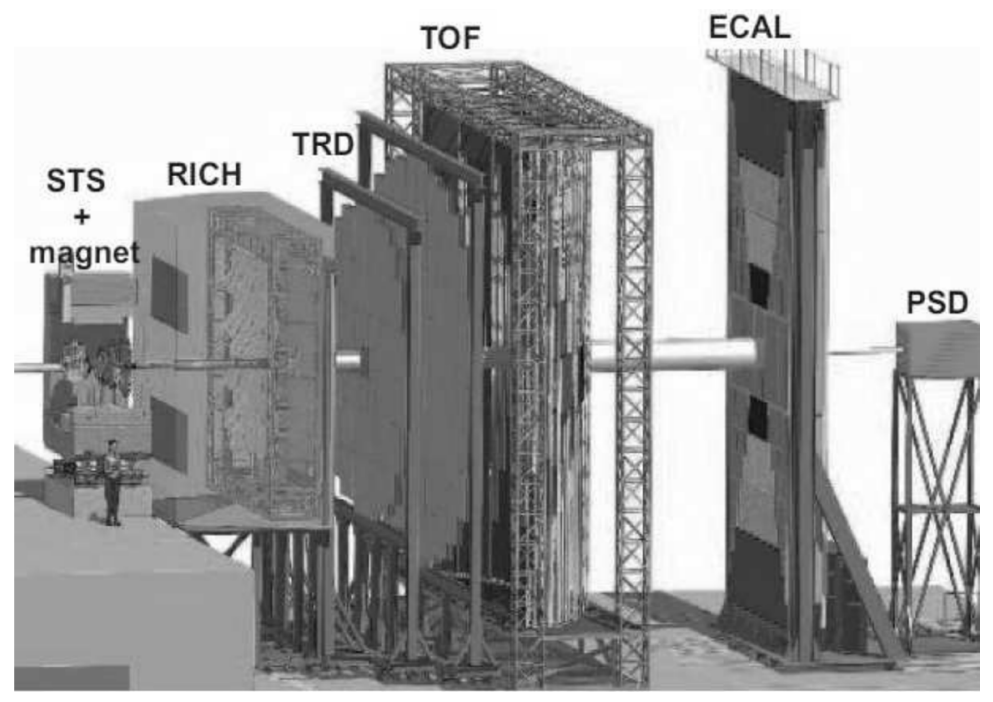

Figure 1. The electron-hadron configuration of the CBM experimental setup

^e-mail: odenisova@jinr.ru 
Behind the target between the poles of the superconducting dipole magnet there is a Silicon Tracking System (STS). The STS detectors are intended to reconstruct trajectories and momenta of charged particles, as well as primary and secondary vertices. The electron-positron identification system includes Ring Imaging CHerenkov (RICH) and Transition Radiation Detectors (TRD). The TRD is also used to reconstruct the trajectories of charged particles registered by the detector. The detector for particle Time-Of-Flight (TOF) measurement is intended for hadron identification. The Electromagnetic CALorimeter (ECAL) serves to identify photons. The Projectile Spectator Detector (PSD) calorimeter determines the reaction plane.

One of the aims of the experiment is to study the production of hypernuclei. The discovery and investigation of new (doubly strange-) hypernuclei will shed light on the hyperon-nucleon and hyperonhyperon interactions. In order to accurately measure the yields of hypernuclei and their lifetime, it is necessary to identify their decay products including ${ }^{3} \mathrm{He}$ and ${ }^{4} \mathrm{He}$.

The electron-hadron configuration of the CBM setup allows to measure the ionisation losses that provides the ability to identify heavy fragments. In this paper the possibility of heavy fragments identification using energy loss method in the STS (Silicon Tracking System) detector was studied.

\section{Energy loss method in the STS detector}

The procedure of $\Delta E / \Delta x$ calculation in the STS is the following [2]

- reconstruct a track;

- each track consists of several hits (reconstructed space points where particle passes through the STS planes), each of which consists of two clusters, there are $2 \times N_{\text {hits }}$ measurements of $\Delta E$ for a track. For each cluster $\Delta E$ is proportional to the total cluster charge.

- $\Delta x$ is calculated from the track inclination;

Fig. 2 presents the dependences of the median value $\Delta E / \Delta x$ from the particle momentum for signal and background events. Signal events consist of ${ }^{3} \mathrm{He},{ }^{4} \mathrm{He}$. They were simulated assuming their thermal distributions. The background events were simulated with UrQMD model, thermal deuterons and tritons. Only tracks with $\Delta E / \Delta x>80000 \mu \mathrm{m}$ are further analysed. The background track suppression factor is 158 .
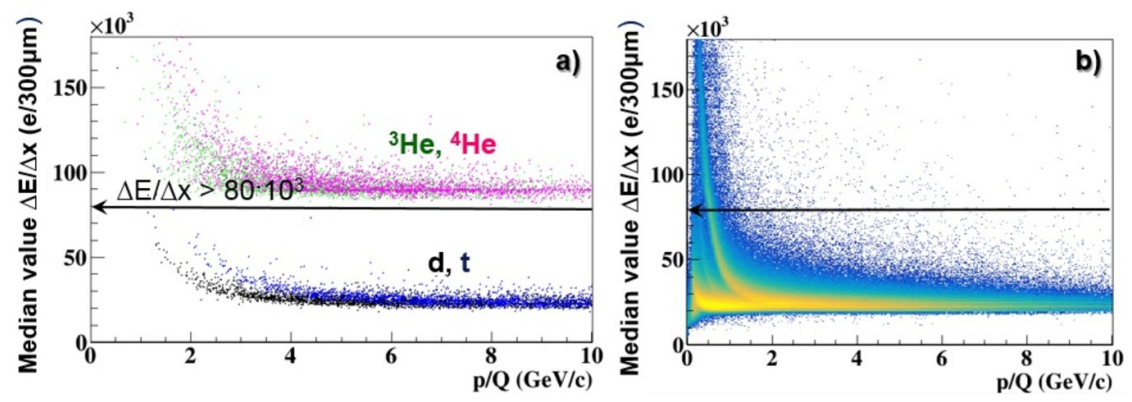

Figure 2. [Color online] The dependences of the median value $\Delta E / \Delta x$ from the particle momentum for a) signal $\left({ }^{3} \mathrm{He},{ }^{4} \mathrm{He}\right)$, thermal deuterons and tritons and b) UrQMD tracks

Although the energy loss method allows to distinguish the doubly-charged particles from the single-ones ( Fig. 2a), there is a large amount of background tracks in the permissible region (Fig. 2b). 


\section{$3 \omega_{n}^{k}$ criterion}

In order to further suppress the background we suggest a method based on the $\omega_{n}^{k}$ goodness-of-fit criterion [3]:

$$
\omega_{n}^{k}=-\frac{n^{k / 2}}{k+1} \sum_{j=1}^{n}\left\{\left[\frac{j-1}{n}-\phi\left(\lambda_{j}\right)\right]^{k+1}-\left[\frac{j}{n}-\phi\left(\lambda_{j}\right)\right]^{k+1}\right\} .
$$

Here $k$ is the criterion degree, $\phi(\lambda)$ is Landau distribution function (which describes $H_{0}$ hypothesis energy losses of the single-charged particles) with a new variable $\lambda$ :

$$
\lambda_{i}=\frac{\Delta E_{i}-\Delta E_{\mathrm{mp}}^{i}}{\xi_{i}}-0.225, \quad i=1,2, \ldots, n,
$$

where $\Delta E_{i}$ is the energy loss in the $i$-th STS layer, $\Delta E_{\mathrm{mp}}^{i}$ is the value of the most probable energy loss, $\xi_{i}=1 / 4.02 \mathrm{FWHM}$ of the distribution of the energy losses for $H_{0}$, and $n$ is the sample size (number of $d E / d x$ values).

Fig. 3 shows the distribution of $\omega_{n}^{k}$ values for a) signal and b) background events, respectively. The $\omega_{n}^{k}$ gives an additional background suppression 1.3, but it does not allow to remove the protons from the analysed samples.
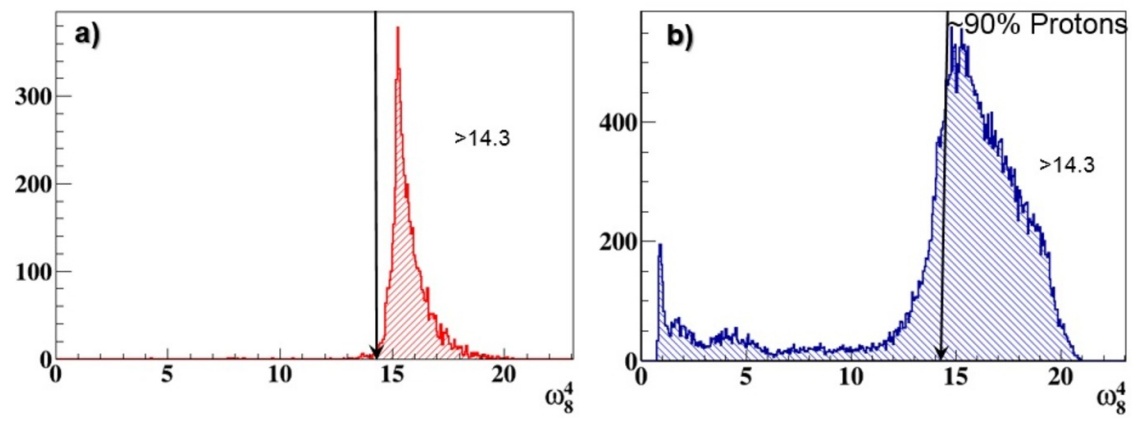

Figure 3. [Color online] Distribution of $\omega_{n}^{k}$ values for a) signal and b) background events

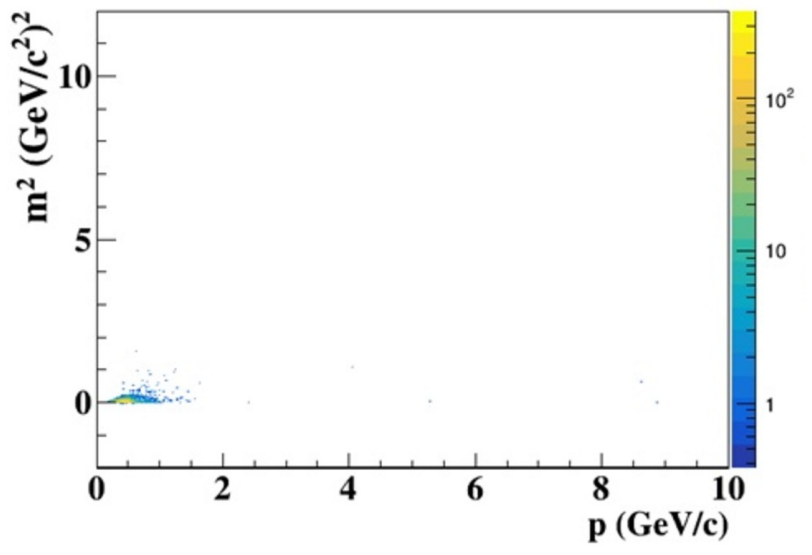

Figure 4. [Color online] The squared mass of charged particles from the background events as a function of the particle momentum 


\section{Proton background suppression with TOF detector}

In order to suppress the protons, the squared mass of the charged particles measured by TOF as a function of the particle momentum is analysed for UrQMD tracks (see Fig. 4 - background events). Small masses indicate that mostly mismatch TOF hits contribute to the sample. The simple linear cut $m^{2}>1\left(\mathrm{GeV}^{2} / c^{4}\right)$ effectively suppresses such background.

Fig. 5 presents the same dependences for the signal events: a) in case when only information from TOF is considered (without STS), and b) TOF after $\Delta E / \Delta x$ and $\omega_{n}^{k}$ criterion cuts in STS.

Therefore, the combination of the TOF and STS allows clearly highlight ${ }^{3} \mathrm{He}$ and ${ }^{4} \mathrm{He}$.
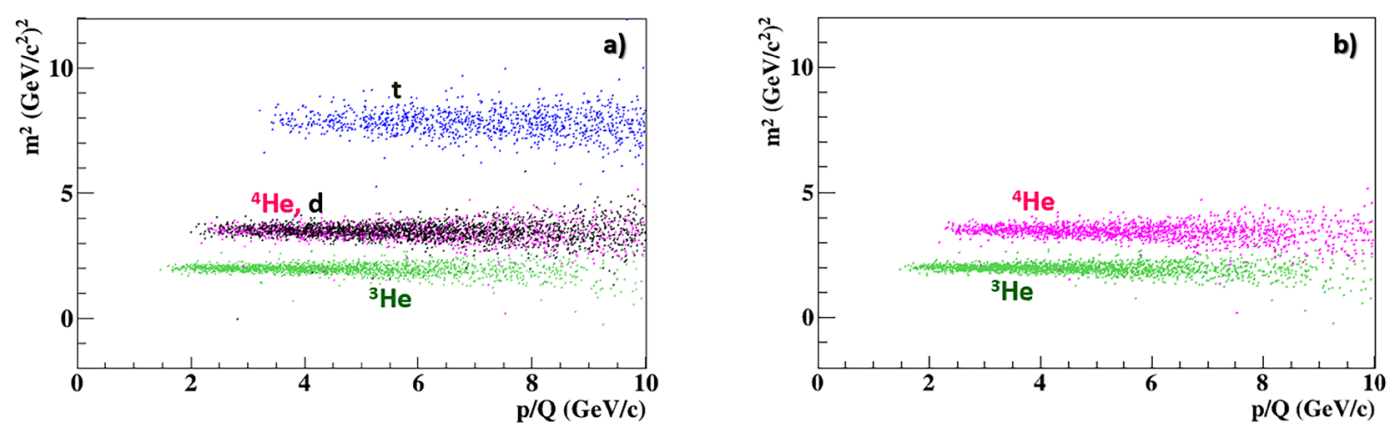

Figure 5. [Color online] The squared mass of charged particles from the signal events as a function of the particle momentum: a) in case when only information from TOF is considered (without STS), and b) TOF after $\Delta E / \Delta x$ and $\omega_{n}^{k}$ criterion cuts in STS

\section{Conclusion}

The $\omega_{n}^{k}$ criterion was successfully adapted for the STS detector to separate the doubly charged particles from singly charged. The combination of the energy loss method with the $\omega_{n}^{k}$ criterion has shown high level of the background suppression without substantiation signal loss. The combination of the information from the TOF and STS detectors allowed to separate ${ }^{3} \mathrm{He}$ and ${ }^{4} \mathrm{He}$ from the deuteron background.

The proposed procedure will be included in the CBM software and applied to the hypernuclei reconstruction.

\section{References}

[1] B. Friman, P. Senger, et al., The CBM Physics Book (Lecture Notes in Physics, vol. 814, 1st Edition, 2011) 960 pp.

[2] H. Malygina, M. Teklishyn, I. Vassiliev, M. Zyzak, CBM Progress Report 2017 (Darmstadt, 2017) p. 160

[3] P.V. Zrelov, V.V. Ivanov, Nucl. Instr. and Meth. in Phys. Res. A 310, 623-630 (1991) 